\title{
Interactions of a Very Long Chain Fatty Acid with Model Membranes and Serum Albumin

\author{
Implications for the Pathogenesis of Adrenoleukodystrophy
}

Jet K. Ho, * Hugo Moser, ${ }^{\ddagger}$ Yasuo Kishimoto, ${ }^{\$}$ and James A. Hamilton ${ }^{*}$

*Department of Biophysics-CABR 302, Boston University School of Medicine, Boston, Massachusetts 02118; ${ }^{\ddagger}$ Johns Hopkins School of Medicine and Kennedy Krieger Institute, Baltimore, Maryland 21205; and ${ }^{\S}$ Department of Neurosciences, Center for Molecular

Genetics, University of California, San Diego, School of Medicine, La Jolla, California 92093-0634

\begin{abstract}
Adrenoleukodystrophy (ALD) is an inherited disorder of fatty acid metabolism marked by accumulation of very long chain saturated fatty acids (VLCFA), especially the 26-carbon acid, hexacosanoic acid (HA), in membranes and tissues. We have studied interactions of ${ }^{13} \mathrm{C}$-enriched $\mathrm{HA}$ with model membranes (phospholipid bilayer vesicles) and bovine serum albumin (BSA) by ${ }^{13} \mathrm{C}$ NMR spectroscopy to compare properties of $\mathrm{HA}$ with those of typical dietary fatty acids. In phospholipid bilayers the carboxyl group of $\mathrm{HA}$ is localized in the aqueous interface, with an apparent $\mathbf{p K}_{\mathrm{a}}$ (7.4) similar to other fatty acids; the acyl chain must then penetrate very deeply into the membrane. Desorption of HA from vesicles $\left(t_{1 / 2}=3 \mathrm{~h}\right)$ is orders of magnitude slower than shorter chain fatty acids. In mixtures of vesicles and BSA, HA partitions much more favorably to phospholipid bilayers than typical fatty acids. BSA binds a maximum of only 1 mole of HA at one binding site. Calorimetric experiments show strong perturbations of acyl chains of phospholipids by HA. We predict that disruptive effects of VLCFA on cell membrane structure and function may explain the neurological manifestations of ALD patients. These effects will be further amplified by slow desorption of VLCFA from membranes and by the ineffective binding to serum albumin. (J. Clin. Invest. 1995. 96:1455-1463.) Key words: lipid bilayers • lipid metabolism • inborn errors - nuclear magnetic resonance $\bullet$ calorimetry $\bullet$ differential scanning $\bullet$ serum albumin • bovine
\end{abstract}

\section{Introduction}

Abnormally high levels of unbranched saturated very long chain fatty acids (VLCFA), ${ }^{1}$ particularly hexacosanoic (C26:0) and

\footnotetext{
Address correspondence to James A. Hamilton, Ph.D., Department of Biophysics-CABR 302, Boston University School of Medicine, 80 East Concord Street, Boston MA 02118. Phone: 617-638-5048; FAX: 617638-4041; e-mail: hamilton@med-biophd.bu.edu

Received for publication 19 October 1994 and accepted in revised form 6 June 1995.

1. Abbreviations used in this paper: ALD, adrenoleukodystrophy; AMN, adrenomyeloneuropathy; DPPC, 1,2-dipalmitoyl-sn-glycero-3-phosphocholine; DSC, differential scanning calorimetry; HA, hexacosanoic acid; NMR, nuclear magnetic resonance; PC, phosphatidylcholine; VLCFA, very long chain fatty acid.
}

J. Clin. Invest.

(c) The American Society for Clinical Investigation, Inc.

$0021-9738 / 95 / 09 / 1455 / 09 \$ 2.00$

Volume 96, September 1995, 1455-1463 tetracosanoic acid (C24:0) fatty acids, are a characteristic feature of X-linked adrenoleukodystrophy (ALD) (1), a genetically determined disorder associated with progressive destruction of myelin and primary adrenocortical insufficiency. The biochemical and metabolic properties of VLCFA have been extensively studied (2). The VLCFA excess in ALD is due to the impaired capacity to form their Coenzyme A derivative ( 3 , 4 ), a process that normally takes place in the peroxisome (5). The putative gene defect has been identified recently (6).

Since the accumulation of VLCFA is the principal and most identifiable biochemical abnormality in ALD, it seems plausible that this change is related to the pathogenesis of the disease. However, at this time there is no proof that this is the case. While correlative data suggest that adrenal insufficiency is a consequence of the accumulation of cholesterol esterified with VLCFA (7), the pathogenesis of the nervous system pathology is not yet understood. Approximately half of the patients succumb in childhood or adolescence to a rapidly progressive destruction of myelin associated with an inflammatory response which involves cytokines (8) and possibly autoimmune mechanisms (9). The inflammatory response is mild or absent in ALD patients who have a milder phenotype, referred to as adrenomyeloneuropathy (AMN), which manifests in adulthood and is compatible with survival to the seventh or eighth decade. The AMN patients show the same degree of impairment of VLCFA degradation and the same degree of VLCFA accumulation as the boys who succumb in childhood $(6,10)$. The VLCFA excess thus is not sufficient as a cause of the rapidly progressive demyelinating process.

Current therapies for ALD include the administration of a mixture of glyceryl trierucate and trioleate oil (also referred to as Lorenzo's Oil), which normalizes the levels of VLCFA in plasma within four weeks $(11,12)$. In spite of this promising biochemical effect, clinical results have been disappointing when the oils were fed to symptomatic patients $(13,14)$. However, a positive result has been obtained in patients in whom therapy was begun before neurological symptoms were present (15), suggesting that the fatty acid abnormality is of pathogenic significance. Up to this time the main support for a direct toxic effect of VLCFA is provided by studies that show that red cell membranes of ALD patients have increased microviscosity and VLCFA content (16) and that addition of VLCFA to adrenocortical cells in vitro increases their membrane microviscosity and impairs their capacity to respond to ACTH stimulation (17).

To investigate further the effect of VLCFA excess on membrane structure and function, we have used biophysical approaches, NMR spectroscopy and differential scanning calorimetry (DSC). ${ }^{13} \mathrm{C}$ NMR spectroscopy has been particularly useful in illuminating properties of fatty acids bound to albumin and to model membranes (18-21). Native fatty acids containing a nonperturbing ${ }^{13} \mathrm{C}$-enriched nucleus in a specific carbon atom 
provide spectroscopic information about the local environment of the fatty acid. Moreover, ${ }^{13} \mathrm{C}$ NMR methods can be used to monitor the movement of fatty acid between binding proteins and membranes and determine the partitioning at equilibrium $(22,23)$. Previous NMR studies have examined fatty acids ranging in chain length from 8 (octanoic acid) to 18 carbons (oleic and stearic acid) (19-25). DSC provides complementary information about interactions of fatty acids with the acyl chains of phospholipids and has been previously used to study saturated fatty acids with chain lengths of 7-24 carbons (26-28).

The present study focuses on a specific VLCFA, the 26 carbon saturated hexacosanoic acid (HA; C26:0), and its interaction with model membranes (phospholipid bilayers) and bovine serum albumin (BSA). HA binds to phospholipid vesicles and exhibits an ionization behavior in the membrane indistinguishable from other fatty acids. However, the acyl chain of HA perturbs the surrounding phospholipids in a manner distinct from other fatty acids. Partitioning to serum albumin from model membranes is not as favorable as its shorter counterparts. In contrast to the multiple binding sites on BSA described previously for fatty acids, only one binding site on albumin is observed for HA. The rate of spontaneous desorption of HA from a phospholipid bilayer transfer is much slower than rates of shorter chain fatty acids. Properties of VLCFA, as elucidated by this study, help explain the pathogenesis of diseases related to these fatty acids.

\section{Methods}

Synthesis of $\left[1-{ }^{13} \mathrm{C}\right]$ hexacosanoic acid. $\left[1-{ }^{13} \mathrm{C}\right]$ Hexacosanoic acid was synthesized as described previously for the synthesis of $\left[2{ }^{2} \mathrm{H}\right]-$ lignoceric acid (29) with some modification. Briefly, $1 \mathrm{mmol}$ (354 mg) of tetracosanol (from NuCheck) was converted to its methanesulfonyl ester by reacting with $200 \mu \mathrm{l}$ of methanesulfonyl chloride in $10 \mathrm{ml}$ of dry pyridine. Slight warming of the mixture was necessary to dissolve tetracosanol. The yield of the ester was $355 \mathrm{mg} .\left[1,3-{ }^{13} \mathrm{C}\right]$ Diethyl malonate $\left(250 \mathrm{mg} ; 99 \%{ }^{13} \mathrm{C}\right.$ enrichment) was obtained from Cambridge Isotope Laboratories and reacted with $61 \mathrm{mg}$ of sodium hydride (supplied from Aldrich as $60 \%$ oil suspension) in $4 \mathrm{ml}$ dry benzene for 30 min. After addition of $140 \mathrm{mg}$ of tetracosanol methanesulfonyl ester in $3 \mathrm{ml}$ of dry benzene, the mixture was refluxed in an oil bath overnight. The reaction product was extracted and partially purified by column chromatography on 7 grams of Florisil (100-200 mesh). The column was first rinsed with $300 \mathrm{ml}$ of hexanes and the product, ethyl $2-\left[{ }^{13} \mathrm{C}\right]-$ carboethoxyl $\left[1-{ }^{13} \mathrm{C}\right]$ hexacosanoate, was eluted with $200 \mathrm{ml}$ of hexaneether $(75-25 \mathrm{vol} / \mathrm{vol})$.

The above product was saponified by refluxing for $4 \mathrm{~h}$ in $5 \mathrm{ml}$ of a mixture of 5 grams $\mathrm{NaOH}$ dissolved in $10 \mathrm{ml}$ of water and $90 \mathrm{ml}$ ethanol. The saponified product was extracted with ether after acidification with $\mathrm{HCl}$. The residue, after evaporation of ether, was decarboxylated by heating in $200^{\circ} \mathrm{C}$ glycerol bath for $10 \mathrm{~min}$. The product was recrystallized from $10 \mathrm{ml}$ hexane and the HA was purified by silica chromatography using a hexane/ethyl acetate/acetic acid gradient with $1 \%$ acetic acid and increasing amounts of ethyl acetate, from 0 to $19 \%$. The following analyses were performed on the purified HA. TLC on silica gel in a hexane/acetic acid/ethyl acetate (80:1:19) solvent system showed $<2 \%$ impurities. Gas chromatography showed no fatty acids of shorter length; in particular, the purified HA was essentially free from contamination by fatty acids with $14-24$ carbons. ${ }^{13} \mathrm{C}$ NMR of the fatty acid detected no contamination of other ${ }^{13} \mathrm{C}$-enriched substances.

Preparation of $P C$ vesicles containing C26:0 fatty acid. Egg PC purchased from Avanti (Lot No. EPC-253) showed one spot by TLC in a hexane, acetic acid, ethyl acetate $(80 / 1 / 19, \mathrm{vol} / \mathrm{vol} / \mathrm{vol})$ solvent systems. The desired amounts of HA and egg yolk phosphatidylcholine (PC) were mixed in chloroform and the solvent removed by a stream of dry nitrogen. The uniform thin film formed on a round bottom flask was then dried under vacuum overnight. In some cases the sample was heated to $85^{\circ} \mathrm{C}$ for one minute before adding buffer to facilitate dispersing. The mixture was vigorously vortexed in $1.8-2.0 \mathrm{ml}$ of $75 \mathrm{mM}$ $\mathrm{KCl}$ containing $10 \% \mathrm{D}_{2} \mathrm{O}$ until evenly dispersed. The sample was then sonicated in a cold water bath for an hour using a Sonifier cell disrupter 350 with a Branson microtip. The clarified suspension was centrifuged for $20 \mathrm{~min}$ to sediment metallic fragments from the sonicator probe and a small amount of undispersed material.

Preparation of BSA solution. Bovine serum albumin (Initial Fractionation by Cold Alcohol Precipitation, Essentially fatty acid free) purchased from Sigma (Lot No. 50H9329) was dissolved in aqueous $75 \mathrm{mM} \mathrm{KCl}$ with $10 \% \mathrm{D}_{2} \mathrm{O}$. The solution was filtered through a $2 \mu \mathrm{m}$ pore filter (Acrodisc by Gelman) pre-rinsed with $\mathrm{KCl}$ solution to remove any glycerol and lipids. The concentration of BSA was determined by UV spectrometry at a wavelength of $279.0 \mathrm{~nm}$.

Transfer of fatty acid from vesicles to BSA. PC vesicles with HA were mixed with aqueous BSA to achieve an equal mass of BSA and PC, and 1.7 mole of fatty acid per mole of BSA. Another sample was prepared with a $2 / 1$ mass ratio of BSA to $\mathrm{PC}$, and a stoichiometry of 0.84 mole of fatty acid per mole of BSA. After acquiring data for the 2/1 BSA/PC sample, BSA was added to increase the BSA/PC ratio to $3: 1$, further reducing the stoichiometry to 0.56 .

The partitioning at equilibrium was quantitated by calculation of the partition coefficient $\left(K_{\mathrm{p}}\right)$ according to the equation:

$$
K_{\mathrm{p}}=\frac{\begin{array}{l}
\text { moles of HA bound to } \mathrm{PC} / \\
\text { moles of PC }
\end{array}}{\begin{array}{c}
\text { moles of HA bound to } \mathrm{BSA} / \\
\text { moles of BSA }
\end{array}}
$$

The moles of PC, BSA, and HA in the sample mixture are known quantities; the moles of HA bound to PC or to BSA were calculated from the relative peak intensities of $\mathrm{HA}$ in the spectrum of the mixture.

NMR Spectroscopy. ${ }^{13} \mathrm{C}$ NMR spectra were acquired at $50.3 \mathrm{MHz}$ on a Bruker WP-200 spectrometer with a broad-band 10-mm probe or at $75 \mathrm{MHz}$ on Bruker AMX-300 spectrometer with a QNP 10-mm probe. The sample temperature was calibrated to $35 \pm 1^{\circ} \mathrm{C}$. Spectra were processed by the NMR1 program from NMR Resources (Tripos, Inc., St. Louis, MO) on a VAX workstation. The NMR titration curve was obtained by titrating the vesicle sample against $\mathrm{KOH}$ and $\mathrm{HCl}$ between $\mathrm{pH} 4.6$ and 9.5. $\mathrm{pH}$ values were measured in the NMR sample tube with a Microelectrodes (Londonderry, $\mathrm{NH}$ ) electrode before and after NMR experiments and differed by $<0.3 \mathrm{pH}$ units; the $\mathrm{pH}$ values after NMR were used for data analyses. NMR chemical shifts were referenced to the fatty acyl $\mathrm{CH}_{3}$ signal of phospholipids ( $14.10 \mathrm{ppm}$ ) and were not dependent on the sequence of obtaining different $\mathrm{pH}$ 's. As in the case of a typical long chain fatty acid in vesicles (21), the vesicle suspension remained translucent throughout the titration.

Calorimetry. HA (Analab, Inc. Lot G1194) was dissolved in warm hexane. Palmitic acid (NuChek Prep, Inc. Lot N-16A-F8-2) and 1,2dipalmitoyl-sn-glycero-3-phosphocholine (DPPC, Avanti Polar Lipids, Lot 160PC-201) were dissolved in chloroform. Palmitic acid or HA was mixed with DPPC at the appropriate mole ratio. At the higher concentrations of HA, additional chloroform was added to prevent precipitation of DPPC. After the solvents were evaporated under $\mathrm{N}_{2}$ gas to form a homogenous mixture, the mixture was placed in vacuum overnight. The dried powder was weighed into a cell with $12 \mu \mathrm{l}$ of water to make hydrated multilayers of DPPC with incorporated fatty acid. DSC experiments were performed on Perkin Elmer DSC-7 calorimeter. Each sample was cycled between 0 and $100^{\circ} \mathrm{C}$ at least three times before data acquisition to ensure homogenity and uniform hydration. At least two heating and cooling cycles were run for each sample. Data was acquired between 0 and $80^{\circ} \mathrm{C}$, with heating and cooling rates of $5^{\circ} \mathrm{C} /$ min. The agreement of the phase transitions of pure DPPC with those in the literature (for example, reference 30 ) showed that all traces of solvents had been removed.

\section{Results}

Titration of HA bound to phospholipid vesicles. To probe interactions of HA with model membranes, a small proportion 

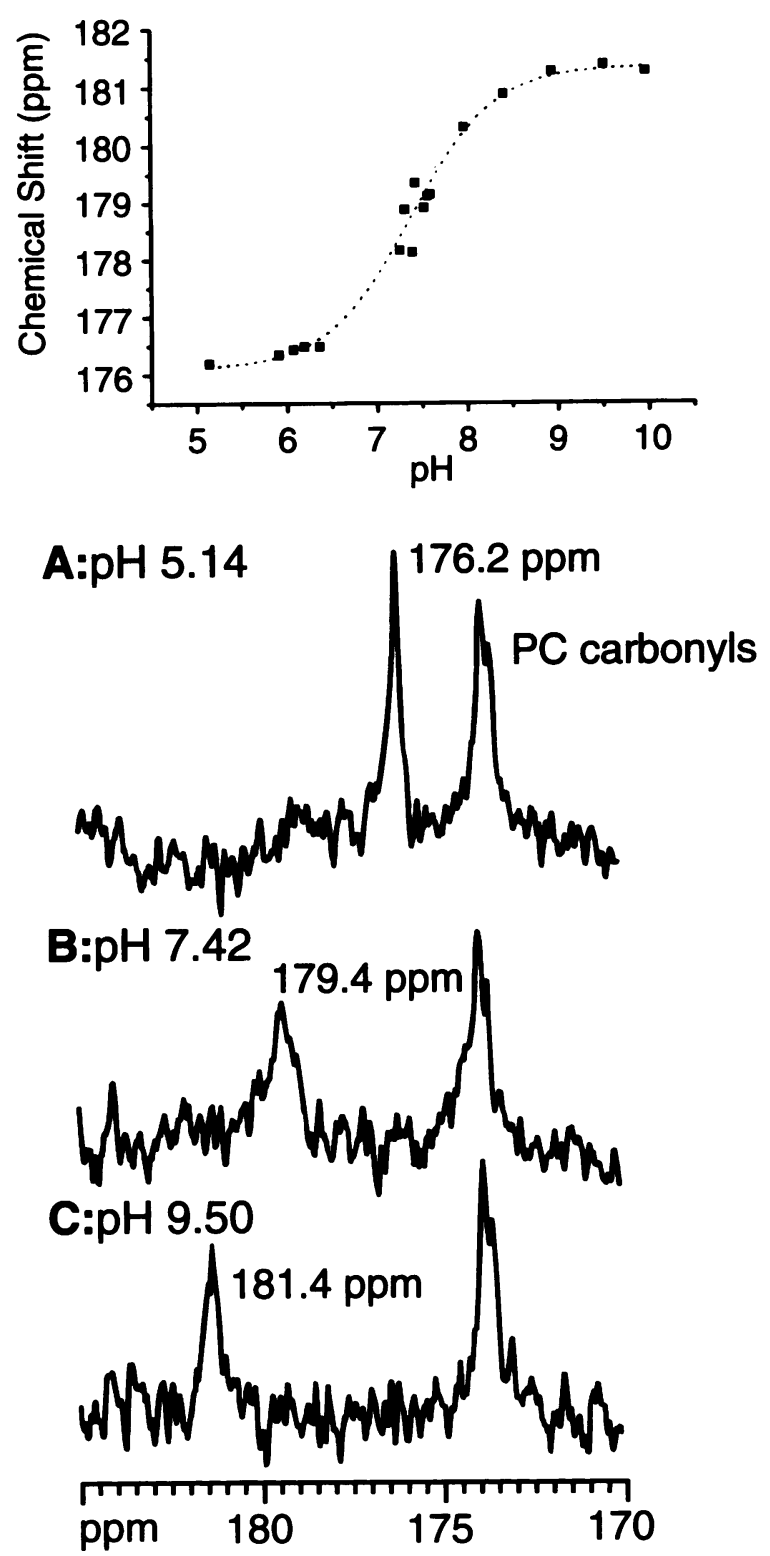

Figure 1. Representative spectra (carboxyl region) at $50.3 \mathrm{MHz}$ of PC vesicles containing ${ }^{13} \mathrm{C}$-enriched $\mathrm{HA}$. The $\mathrm{PC}$ carbonyls at 173.9 and $173.6 \mathrm{ppm}$ represent the carbonyls on the outer and inner leaflets of the vesicles, respectively (22). The single peak at higher ppm represents HA bound to the PC vesicles at selected $\mathrm{pH}$ values: $(A) \mathrm{pH} 5.14 ;(B)$ pH 7.42; $(C) \mathrm{pH} 9.50$. Spectra were obtained with $16 \mathrm{~K}$ data points and a pulse interval of 2.0 seconds and processed after 2000 spectral accumulations. (TOP) Plot of the chemical shift of the carboxyl carbon of HA bound to PC vesicles versus $\mathrm{pH}$. The dotted line shows a theoretical prediction of the chemical shifts by the Henderson-Hasselbach equation (25). The minimum and maximum chemical shifts were 176.2 and $181.4 \mathrm{ppm}$, respectively. The $\mathrm{pK}_{\mathrm{a}}$ determined by curve fitting is 7.4 .

( $\sim 2 \mathrm{~mol} \%$ with respect to phospholipid) of ${ }^{13} \mathrm{C}$-carboxyl-enriched HA was incorporated into egg PC unilamellar vesicles by cosonication of $\mathrm{HA}$ and PC. ${ }^{13} \mathrm{C}$ NMR spectra of the PC vesicles with $\mathrm{HA}$ were obtained at different $\mathrm{pH}$ values. Selected spectra of the carbonyl region (Fig. 1) show a resonance from the ${ }^{13} \mathrm{C}$-enriched $\mathrm{HA}$ in addition to the natural abundance carbonyl signals from phospholipid. This signal reflects HA bound to vesicles, since unbound $\mathrm{HA}$ would be insoluble in the aqueous buffer and yield a very broad (if observable) NMR peak
(19). Fig. 1 shows that with decreasing $\mathrm{pH}$, the carboxyl peak of HA shifted upfield, whereas the chemical shifts of non-titratable phospholipid carbonyls were unchanged. The maximum downfield (complete ionization) and upfield (complete protonation) chemical shifts were 181.4 and $176.2 \mathrm{ppm}$, respectively, the same as those observed for similar amounts of oleic or palmitic acid in PC vesicles $(21,22)$. The plot of the carboxyl chemical shift versus pH (Fig. 1, top) is sigmoidal in shape, as in the case of shorter chain FA. The data are well represented by the Henderson-Hasselbach equation, and the apparent $\mathrm{pK}_{\mathrm{a}}$ of the $\mathrm{HA}$ in the PC vesicles was calculated to be 7.4. The $\mathrm{pK}_{\mathrm{a}}$ for oleic or palmitic acid titrated under similar conditions in PC vesicles is $7.6(21)$.

Binding of HA to BSA. Since albumin is the major transport vehicle in plasma for fatty acids and both delivers fatty acids to and removes them from cell membranes, it was of interest to study binding of HA to albumin. However, the extremely low water solubility of HA was an obstacle to making fatty acid/albumin complexes. For long chain fatty acids (12-18 carbons), which have a poor solubility at $\mathrm{pH} 7.4$, a soluble (micellar) soap solution can be prepared at high $\mathrm{pH}$ and injected into an aqueous albumin solution at $\mathrm{pH} 7.4$; rapid and quantitative binding to albumin is observed with such a protocol (19, 24). In contrast, we were not able to prepare a soluble solution of HA in the soap form at any desired concentration. We also attempted to make albumin complexes with VLCFA by mixing aqueous BSA with organic solvent containing HA. Similar two phase systems have been used to determine binding affinities of albumin for typical dietary fatty acids (31). Although the solubility of HA in hexane was adequate, binding to BSA was not observed when HA in hexane was injected into an aqueous BSA solution. In comparison, similar experiments with oleic acid showed ${ }^{13} \mathrm{C}$ resonances characteristic of fatty acids bound to albumin (for example see reference 19). Delivery of HA to aqueous albumin via other organic solvents also proved to be unsuccessful.

To facilitate binding of HA to albumin we used phospholipid bilayers as a vehicle for solubilizing the fatty acids. Under appropriate conditions, fatty acids will transfer from vesicles to albumin, and the partitioning at equilibrium can be measured quantitatively by ${ }^{13} \mathrm{C}$ NMR $(22,23)$. With this protocol fatty acid is delivered to albumin in a more physiologically relevant manner compared to procedures utilizing organic solvents. Previous studies with fatty acids having chain lengths of 8-18 carbons found rapid equilibration of fatty acids between phospholipid vesicles and albumin, so that spectra remained unchanged with time $(22,23)$. For HA we observed transfer to BSA but found that equilibrium was not reached for several hours, and it was possible to obtain time-dependent spectra showing the kinetics of equilibration.

Fig. 2 illustrates spectra at different time intervals for a sample comprised of egg PC vesicles with $2 \mathrm{~mol} \% \mathrm{HA}$ as donor particles and (fatty acid-free) BSA as an acceptor. HA bound to $\mathrm{PC}$ vesicles at $\mathrm{pH} 7.4$ (Fig. $2 \mathrm{~A}$ ) is represented by the peak at $178.0 \mathrm{ppm}$, as in Fig. 1. After addition of BSA, two new weaker signals were seen in the carbonyl region at $181.1 \mathrm{ppm}$ and $182.7 \mathrm{ppm}$. The peak from HA bound to the vesicles was still relatively intense at the first time point $(0-1 \mathrm{~h}$; Fig. $3 B)$, but decreased with time (Fig. 3, $C$ and $D$ ). The peak at 181.1 ppm from glutamate carboxyl groups of BSA (19) remained constant in intensity over time, as expected. The most downfield signal (182.7 ppm) exhibited a constant chemical shift and a progressively increasing intensity until a maximum intensity 


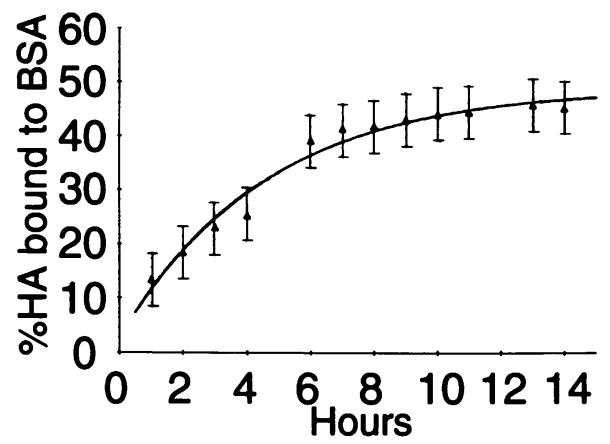

A:HAVC

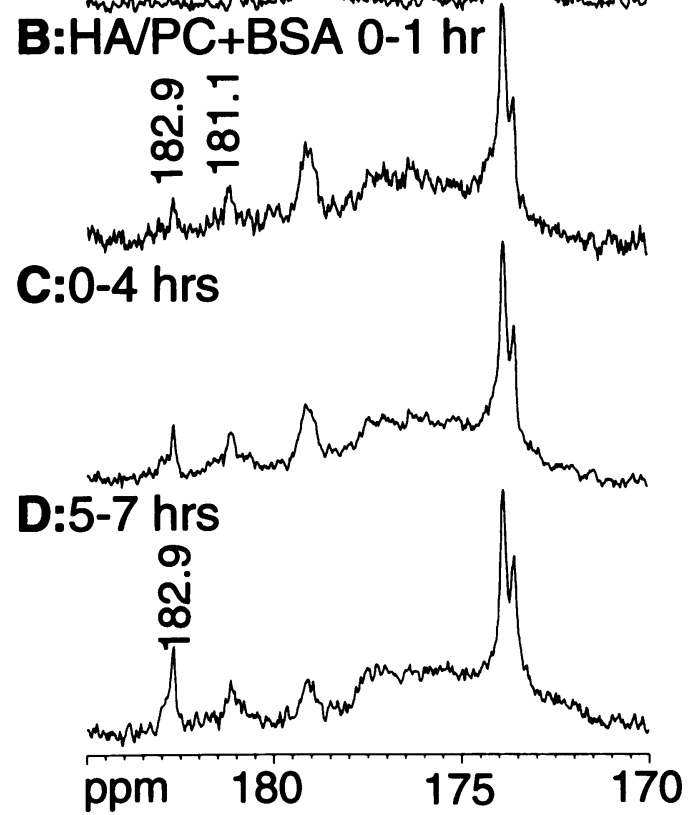

Figure 2. The carboxyl and carbonyl region of the ${ }^{13} \mathrm{C}$ NMR spectrum at $75 \mathrm{MHz}$ illustrating the time dependence of $\mathrm{HA}$ transfer from PC vesicles to BSA. Spectra were obtained for PC vesicles with $\mathrm{HA}$ before $(A)$ and after $(B-D)$ addition of fatty acid-free BSA. Note that the PC carbonyl peaks were unchanged throughout these experiments, reflecting the constant amount of PC and the nontitratability of the carbonyl groups. Spectrum A was obtained after 1800 spectral accumulations and shows HA bound to PC at the expected chemical shift (178.0 ppm) for $\mathrm{pH}$ 7.41. Integration of the peak intensity of the HA carboxyl relative to the PC carbonyls showed good agreement with the expected intensity for $2 \mathrm{~mol} \% \mathrm{HA}$ after correction for the difference in ${ }^{13} \mathrm{C}$ abundance. In spectrum B the peak at $178 \mathrm{ppm}$ decreased in intensity, and two new peaks ( 181.1 and $182.87 \mathrm{ppm}$ ) appeared. The peak at $181.1 \mathrm{ppm}$ represents glutamate carboxyls of BSA and remains constant with time. Spectrum $C$ represents data for the first 4 hours $(7200$ spectral accumulations) after adding BSA, and shows an increased intensity in the signal at $182.7 \mathrm{ppm}$ and a decreased intensity in the signal at $178.0 \mathrm{ppm}$. Spectrum D represents data obtained between the 5th and the 7th hour of incubation ( 3600 spectral accumulations). Subsequent spectra showed very small time-dependent changes in peak intensities, indicating that spectrum $\mathrm{D}$ represents equilibrium partitioning (see text). All spectra were acquired at $35^{\circ} \mathrm{C}$ with a 2.0 second pulse interval and $16 \mathrm{~K}$ time domain points. (Top) Plot of the normalized intensity of the signal was reached at 7-8 h. The intensity changes as a function of time (Fig. 2, top) were well described by a single exponential function with $t_{1 / 2}=3.2 \mathrm{~h}$. The peak at $182.7 \mathrm{ppm}$ is in the spectral region characteristic of long chain fatty acids bound to albumin (20). Spectral simulation showed a linewidth of $\sim 10$ $\mathrm{Hz}$, similar to the linewidth for peaks representing oleic acid in individual sites on BSA [19, 21]. As NMR signals for unbound fatty acid, if present, would be broad and appear at a different chemical shift $(19,32)$, it was concluded that this new signal represents HA bound to BSA.

The above transfer experiment was carried out with a mass ratio of $2 \mathrm{BSA} / \mathrm{PC}$ and a stoichiometric mole ratio of $0.84 \mathrm{HA} /$ BSA. The same protocol was repeated with equal masses of PC and BSA, the ratio used in analogous studies with other fatty acids $(22,23)$, and a stoichiometric ratio of $1.7 \mathrm{HA} / \mathrm{BSA}$. Under these conditions slow transfer (over hours) of HA from PC was also observed, but maximal transfer of HA to BSA was significantly lower (20\%, Fig. $3 A$ ) compared to the $2 / 1 \mathrm{BSA} /$ PC sample ( $45 \%$, Fig. $3 B$ ). The smaller changes in peak intensities precluded quantitative analysis of the transfer kinetics. To investigate further the partitioning as a function of BSA/PC, BSA was added to the $2 / 1 \mathrm{BSA} / \mathrm{PC}$ to make a $3 / 1$ ratio (Fig. $3 C$ ). An increased partitioning of HA to BSA was observed, but even at this higher BSA/PC some HA was bound to PC vesicles. Note that in Fig. 3 that intensities of the signals at $181.1 \mathrm{ppm}$ and the broad carbonyl envelope increase relative to the PC carbonyls; both signals are from BSA and reflect the increasing proportion of BSA in the mixture.

Previous studies have shown that the partitioning of fatty acids (both medium and long-chain) between albumin and PC bilayers is $\mathrm{pH}$-dependent $(22,23)$. Because small changes in $\mathrm{pH}$ below 7.4 can alter partitioning significantly, we investigated HA partitioning under slightly acidic conditions. Fig. 4 compares spectra (carbonyl region) of a mixture of HA, PC vesicles, and $\mathrm{BSA}$ at $\mathrm{pH} 7.6$ and 6.3 after equilibrium partitioning was reached. At $\mathrm{pH} 7.6$ most of the HA was bound to BSA (peak at $182.7 \mathrm{ppm}$ ) and the minority to vesicles (smaller peak at $179.1 \mathrm{ppm}$ ). When the $\mathrm{pH}$ of the solution was decreased to 6.3 , the peak representing the binding to BSA (182.7 ppm) diminished greatly in intensity, and a strong signal at 176.5 ppm was observed. The latter peak is at the chemical shift of HA bound to PC vesicles at pH 6.3 in the absence of BSA (Fig. $1)$. Thus HA, like other fatty acids $(22,23)$, partitions more favorably to PC vesicles than to BSA in a slightly acidic environment. The partitioning was entirely reversible when the $\mathrm{pH}$ of the solution was adjusted back to neutral.

DSC results. The NMR studies of carboxyl-labeled HA in PC bilayers provided important information about the fatty acid headgroup region, but $\mathrm{HA}$ with ${ }^{13} \mathrm{C}$ labels in other positions was not available. To focus on acyl chain interactions of HA with phospholipids and to explore the possibility that HA causes changes in membrane structural organization, calorimetry experiments were performed with the phospholipid bilayers. In these experiments, egg PC is not a useful model membrane because the phase transition occurs near the freezing point of water, and DPPC, a disaturated phospholipid which serves as

of HA bound to BSA versus time. The percentage of HA transferred is determined by the area of the peak at $182.7 \mathrm{ppm}$ divided by the sum of the peak areas of this peak and the peak representing HA bound to $\mathrm{PC}$ vesicles. 


\section{A:1 BSA / 1 PC}

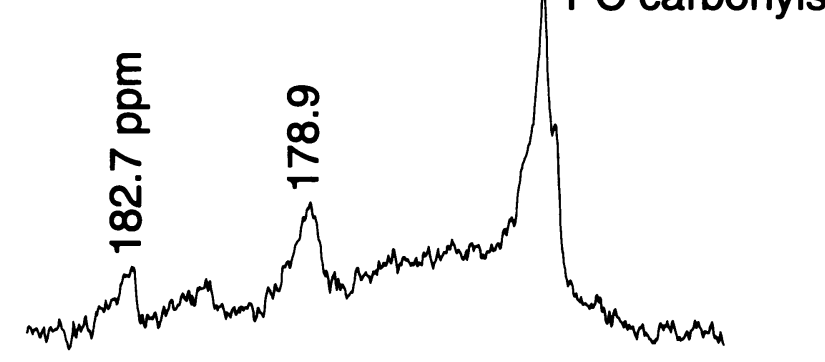

\section{B:2 BSA / 1 PC}
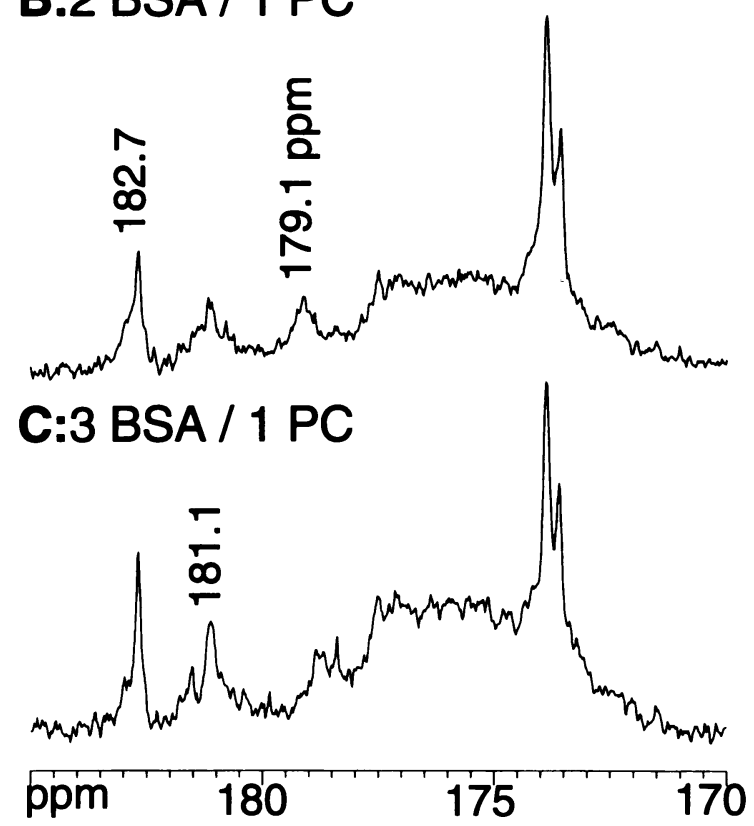

Figure 3. The carbonyl and carboxyl region of the ${ }^{13} \mathrm{C}$ spectrum of an aqueous mixture of $\mathrm{HA}, \mathrm{BSA}$, and $\mathrm{PC}$ vesicles $(\mathrm{pH}=7.4-7.5)$ illustrating partitioning of HA between $\mathrm{PC}$ and BSA at various ratios: $(A) 1$ BSA/1 PC (wt/wt); (B) 2 BSA/1 PC; (C) 3 BSA/1 PC. All spectra were obtained after equilibrium was reached (i.e., no changes with time were observed in the NMR spectra). The peak at $182.7 \mathrm{ppm}$ increased in intensity with increasing BSA/PC, as expected from a simple chemical equilibrium. At 3/1 BSA/PC most of the HA was partitioned to BSA. The peak at $181.1 \mathrm{ppm}$ from the glutamates of BSA increased in intensity as the BSA/PC ratio increased, whereas the peak illustrating PC carbonyls remained constant. Spectra were obtained at $35^{\circ} \mathrm{C}$ with a 2-s pulse interval with $16 \mathrm{~K}$ time domain points after 12,000 spectral accumulations $(A), 7,200$ accumulations $(B)$, and 10,000 accumulations (C).

a standard model for investigating the effects of additives on membrane organization [e.g., 26-28], was used. Fig. 5 compares the behavior of DPPC with a typical dietary fatty acid, palmitic (16:0), or with HA (26:0). Upon heating, pure DPPC shows a small endothermic transition centered at $37^{\circ} \mathrm{C}$ and a larger transition at $42^{\circ} \mathrm{C}$ (bottom trace, panels $A$ and $B$ ). These well-studied transitions represent a phase change first from a gel phase to a rippled gel phase and then to a liquid crystalline transition (30). The major transition represents primarily a change in the acyl chain packing from an ordered to a disordered state. The DSC experiment, as performed here, monitors the effects on phospholipid acyl chain packing of molecules that incorporate into the bilayer structure. Calorimetry experiments carried out on DPPC with varying low proportions of palmitic

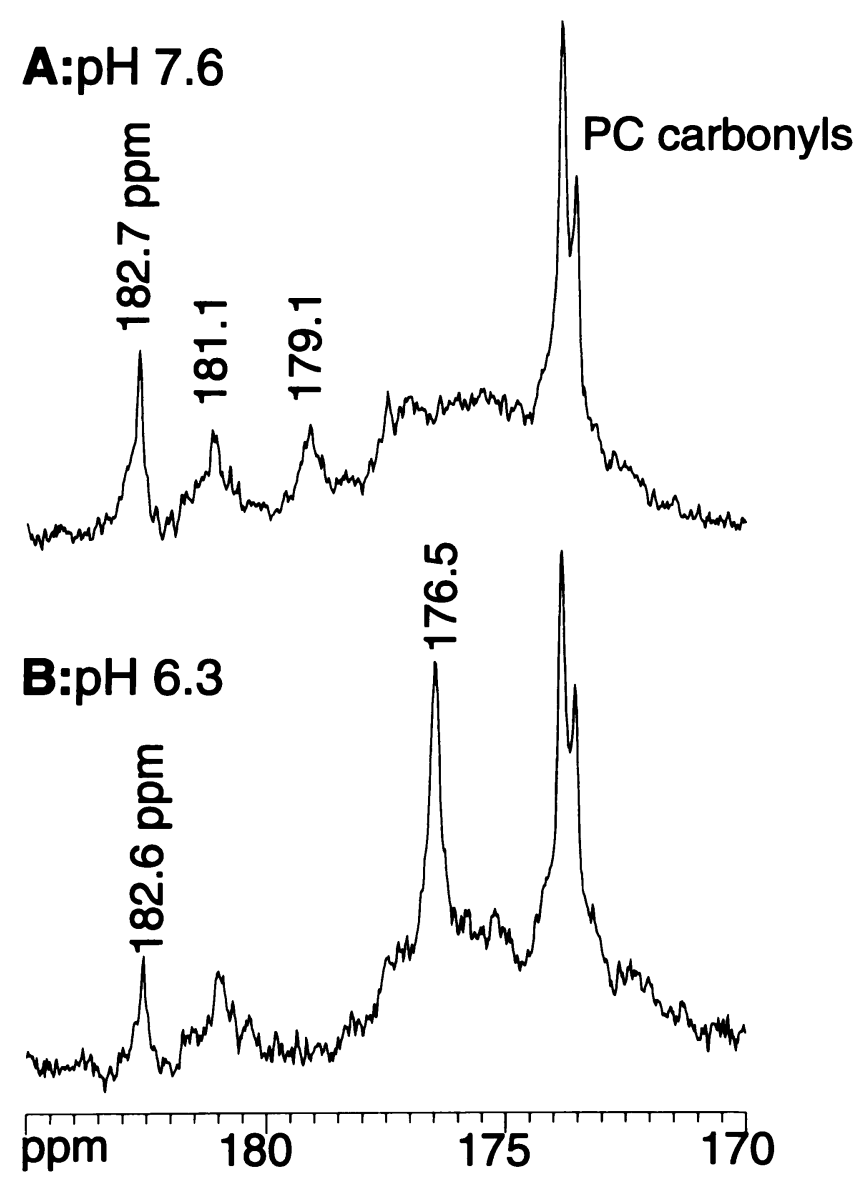

Figure 4. The carboxyl and carbonyl region of the ${ }^{13} \mathrm{C}$ spectrum of an aqueous mixture of HA, BSA and PC vesicles illustrating the $\mathrm{pH}$ dependence of partitioning of HA between PC vesicles and BSA. Spectrum A shows that at $\mathrm{pH} 7.6$, most of the $\mathrm{HA}$ is partitioned to $\mathrm{BSA}$, as reflected in the intense peak at $182.7 \mathrm{ppm}$ for HA bound to BSA. Spectrum B was obtained at $\mathrm{pH} 6.3$ after addition of $\mathrm{HCl}$ to the mixture in A. Most of the HA is partitioned to vesicles, indicated by the increased intensity of the peak at $176.5 \mathrm{ppm}$, reflecting bound to PC vesicles. Spectra were obtained at $35^{\circ} \mathrm{C}$ with a 2.0 -s pulse interval and $16 \mathrm{~K}$ time domain points and processed after 9000 scans $(A)$ and 5400 scans $(B)$.

acid (Fig. $5 \mathrm{~A}$ ) first show the effects of abolishing the pretransition, broadening the main transition, and shifting the midpoint of the main transition to slightly higher temperature. These results indicate that palmitic acid mixes uniformly with the DPPC and has small effects that are transmitted to the bulk of the DPPC molecules. At 10 mole\% palmitic acid a second transition at a higher temperature is observed. At higher palmitic acid, this transition grows at the expense of the lower transition and shifts to higher temperature. This new exotherm is explained by phase separation of fatty acid/phospholipid complexes with a higher melting point from pure DPPC. The described behavior of palmitic acid is in essential agreement with previous reports $(26)$.

The DSC results for HA (Fig. $5 B$ ) are significantly different from that of palmitic acid. At 5-7.5 mole\% HA the small pretransition shifts to higher temperature and then broadens beyond detection, and the main transition broadens but does not shift. At 10-15 mol\% HA, a very broad higher temperature transition, which is complete at $55^{\circ} \mathrm{C}$, becomes apparent, and a sharper lower temperature transition centered at $40^{\circ} \mathrm{C}$ is present. 

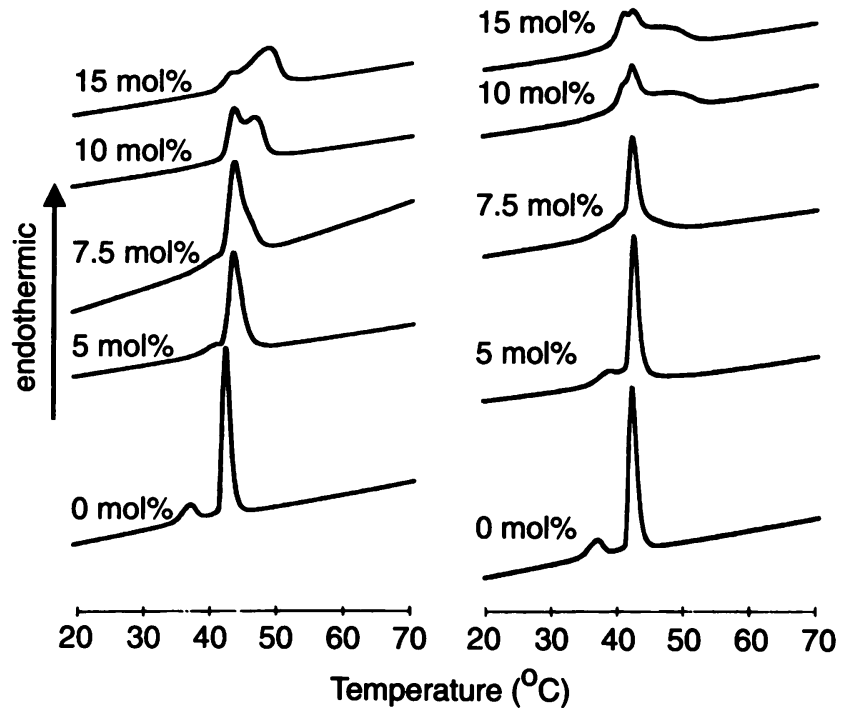

Figure 5. Heating curves for palmitic acid $(A)$ and HA $(B)$ in DPPC multilayers at various mole ratios, from 5-15 mol\%. The bottom trace in each panel is DPPC without added fatty acid.

Although the broad transition is not seen at 5 and $7.5 \% \mathrm{HA}$, it is reasonable to assume that it is present but not visible because the area under the major transition (representing the enthalpy of that transition) is slightly decreased. Previous calorimetry studies of DPPC found that saturated fatty acids with 7-10 carbons decrease, whereas fatty acids with 12-16 carbons increase the main transition temperature (28). It was reported that saturated fatty acids with 20-24 carbons did not alter the main transition temperature, but calorimetry traces were not provided (28). The behavior of HA is therefore unique among all fatty acids reported thus far.

\section{Discussion}

Effect of HA on membrane structure and function and binding of HA to albumin. For the NMR studies presented here, the carboxyl carbon of HA was chosen as the site of ${ }^{13} \mathrm{C}$ enrichment because the chemical shift of this carbon is very sensitive to the surrounding environment. The ${ }^{13} \mathrm{C}$ NMR results demonstrated binding of $\mathrm{HA}$ to model membranes and to albumin. Because of the different molecular environments of HA in membranes and albumin, it was possible to monitor transfer of HA between these two environments in a direct and noninvasive way.

The ionization of the carboxyl group of HA in PC vesicles was not anomalous, showing an apparent $\mathrm{pK}_{\mathrm{a}}$ of 7.4. Moreover, titration data for HA were nearly identical in all respects (minimum and maximum chemical shifts, linewidths and apparent $\mathrm{pK}_{\mathrm{a}}$ ) with data for fatty acids with chain lengths of 14-18 carbons $(18,21,22)$. Thus, the molecular environment of the carboxyl group of HA in phospholipid bilayers is indistinguishable from that of shorter chain fatty acids. Specifically, the carboxyl group must be located at the aqueous interface, in an average position similar to that of other fatty acids. With the carboxyl group thus localized, the long acyl must penetrate deeply into the egg PC bilayer comprised primarily of 16- and 18-carbon chains, chain lengths typical of most cell membranes.

\section{Schematic of C26:0 in PC Bilayers}

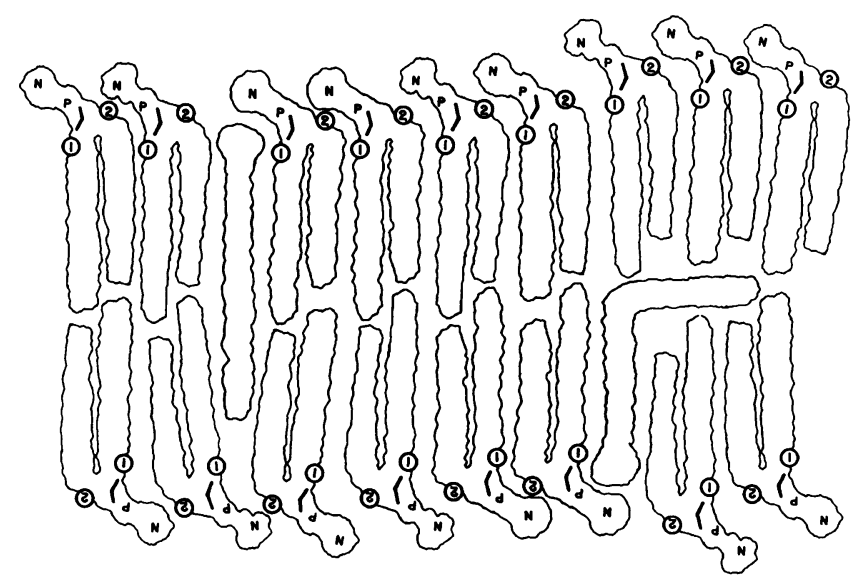

Figure 6. Schematic diagram representing possible binding modes of HA molecules in a phospholipid bilayer. The acyl chains of the phospholipid (PC) have 16 (sn-1) and 18 (sn-2) carbons. The molecules could fit linearly and penetrate about halfway through the opposing phospholipid monolayer or could bend in the middle of the bilayer. Our results localize the carboxyl group at the aqueous interface but do not definitively distinguish different configurations of the acyl chain.

The DSC results showed that HA has a greater tendency than palmitic acid to induce formation of domains in which the acyl chains of DPPC become more rigidified than the bulk phase. At low concentrations, palmitic acid mixed with DPPC and slightly affected the behavior of the entire DPPC matrix, raising the transition temperature. Palmitic acid at higher concentrations also induced phase separation into higher melting domains, but the domains with HA were even higher melting and therefore more ordered. A destabilizing effect is found for HA but not for palmitic acid, as evidenced by a lower temperature transition, and occurred simultaneously with the stabilizing effect. These seemingly contradictory effects can be explained by the deep penetration of $\mathrm{HA}$ into the bilayer.

Fig. 6 shows a schematic diagram based on NMR and calorimetric results of how HA might interact with a phospholipid bilayer in a membrane. Since the NMR titration data show that the carboxyl group is localized in the interfacial region, the terminal region of HA will penetrate between the acyl chains of phospholipids on the opposing leaflet or bend in the middle of the bilayer, as illustrated. The DSC results showing a rigidifying effect of HA on neighboring phospholipid acyl chains would most likely be accomplished with the acyl chain lying fairly straight, which would promote ordering of the palmitate chains of the adjacent DPPC molecules all the way to the end of the chain. This conformation would produce a stronger ordering than a typical long chain saturated fatty acid such as palmitic or stearic. The tendency of HA to produce disordering simultaneously with ordering could be explained by the penetration of the same molecule part way into the acyl chains on the opposing leaflet and acting like a small hydrocarbon in this aspect. In the alternative arrangement depicted in Fig. 6, the presence of a sharp bend in the HA chain to allow the terminal portion to reside in the methyl interior region of the bilayer would likely cause disordering near the ends of all the nearby DPPC chains. The DSC analysis might in this case show only a decrease in the transition temperature reflecting only a disordering effect. Both models of the intercalation of HA into bilayers predict 


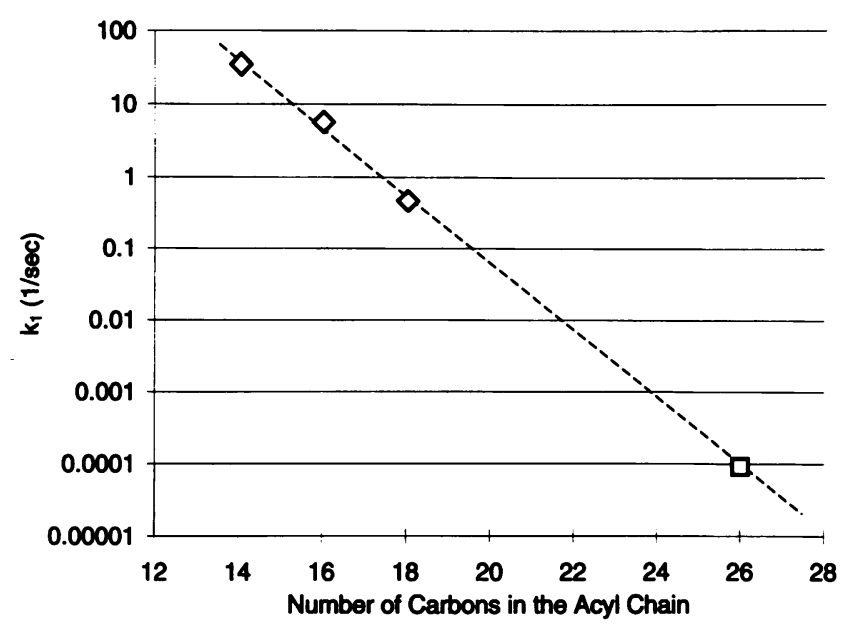

Figure 7. The rate constant for desorption of saturated fatty acids from phospholipid vesicles as a function of chain length. The linear relationship on a semi-log plot of these two variables indicates that the rate of desorption decreases exponentially with increased chain length. The data for myristic, palmitic, and stearic acids $(\diamond)$ are from reference 32 and the data for HA ( $\square$ ) from this study.

another perturbation of bilayer organization, which is the distortion of the phospholipid interface. From steric considerations, the HA is likely to force phospholipid molecules on the opposing leaflet (and possibly the same leaflet) to change their packing in the interface. This effect could alter the binding of proteins and surface antigens and could augment the potential effect of localized ordering caused by HA. The major effect seen by DSC, ordering of phospholipids, is consistent with previous studies showing that VLCFA cause increased microviscosity in cell membranes $(16,17)$.

As a model system for the transfer of VLCFA between membranes and soluble fatty-acid binding proteins, we studied the more complex aqueous mixture of lipid bilayers, albumin, and HA. This model eliminates energy-dependent and (possibly) protein-mediated uptake of fatty acid and allows focus on physico-chemical partitioning. Our studies with HA yielded several important conclusions: (a) the rate of transfer of HA from phospholipid membranes is much slower than rates for typical dietary fatty acids; $(b)$ the affinity of BSA for HA is lower than that for shorter chain fatty acids; $(c)$ HA resides in a single binding site on albumin, and $(d)$ acidic $\mathrm{pH}$ conditions favor the partitioning of HA to membranes, as found for other fatty acids.

From the kinetic analysis of the data for HA desorption from vesicles (Fig. 4), the half time for HA transfer was found to be $3.2 \mathrm{~h}$. The corresponding observed rate constant $\mathrm{k}_{\mathrm{obs}}$ is 6 $\times 10^{-5} \mathrm{~s}^{-1}$, and the pseudo first order rate constant $\left(\mathrm{k}_{1}\right)$ calculated from $k_{o b s}$ and the partition coefficient (see below) is 3 $\times 10^{-5} \mathrm{~s}^{-1}$. The rate constants for transfer of typical dietary fatty acids from phospholipid vesicles to albumin, as determined by fluorescence (32), are orders of magnitude higher [myristate $\left(35.2 \mathrm{~s}^{-1}\right)$; palmitate $\left(5.6 \mathrm{~s}^{-1}\right)$; stearate $\left(0.46 \mathrm{~s}^{-1}\right)$ and oleate $\left(3.45 \mathrm{~s}^{-1}\right)$ ]. Thus, desorption of HA from phospholipid vesicles is $10^{5}-10^{6}$ times slower. Moreover, when the pseudo first order rate constants for saturated fatty acids are plotted as a function of chain length (Fig. 7), the fluorescence data fit closely to a straight line on a semi-log plot, and the rate constant for $\mathrm{HA}$ is well predicted. The observed first order kinetics suggests that the fatty acids transfer by a solubilized form rather than by collision. It is interesting to note that cholesterol, which has 27 carbons and a single $\mathrm{OH}$, desorbs from $\mathrm{PC}$ vesicles with a rate very similar to that of HA (33).

Although the rate of spontaneous desorption from a lipid membrane is slow, it still may be fast enough to allow movement of HA from the plasma membrane to intracellular sites and permit utilization in normal individuals. (We have found, by use of a new fluorescence method [34] that the spontaneous transbilayer movement of HA in PC vesicles is fast $\left[t_{1 / 2}<1 \mathrm{~s}\right]$ [Zhang, F., F. Kamp, and J. A. Hamilton, unpublished observations]. Therefore, HA, like other fatty acids, can in principle diffuse across a membrane without a protein transporter.) However, in ALD patients where utilization is blocked, and higher levels of VLCFA accumulate, the slow desorption of VLCFA from membranes may augment their deleterious effects for at least two reasons. First, the lifetime in a binding site on the membrane is much longer than lifetimes of shorter chain fatty acids, and effects in a specific environment will be more prolonged. Fatty acids are now known to affect biological activities, such as $\mathrm{K}^{+}(35)$ and $\mathrm{Ca}^{++}(36)$ channel opening, to act as signals (37), and to alter intracellular $\mathrm{pH}(38)$. The temporal relationship between fatty acids and membrane proteins may be significantly altered in the case of VLCFA. Secondly, the VLCFA would appear to be a more stable target for enzymatic activation to acyl CoA, and may be more likely to be esterified to lipids or proteins and become an even more long-lived membrane perturbant. In brain white matter HA accumulates primarily in the form of specific esterified lipids. Phosphatidylcholine is 40-fold enriched in HA in ALD patients compared to normals, whereas other glycerophospholipids do not show enrichment in VLCFA (39). In ALD white matter HA accounted for $3.9-6.2 \%$ of total fatty acids in the phosphatidylcholine fraction and between 0.1 and $1.5 \%$ in the phosphatidylserine-ethanolamine and inositide fractions compared to $\sim 0.1 \%$ in all of these fractions in controls. Cholesteryl esters are an extremely minor lipid component in normals but accumulate to high levels in ALD brain white matter and are esterified to a significant extent with VLCFA (40). The fatty acids esterified to myelin proteolipid protein, a double acylated protein which constitutes the major protein of the CNS myelin sheath, also show enrichment in VLCFA (41).

The ${ }^{13} \mathrm{C}$ NMR spectra of the mixture of vesicles, albumin and HA obtained at equilibrium also show important differences compared to spectra of typical fatty acids. The partitioning of HA to BSA in the presence of membranes is significantly decreased, as shown by comparison with previous data obtained under similar conditions $(22,23)$. For example, for an equal weight ratio of $\mathrm{BSA}$ and $\mathrm{PC}$ at $\mathrm{pH} 7.4$, almost all of the oleic acid is bound to BSA, and the calculated partition coefficient $K_{p}$ (Methods) is 0.002 . For the same BSA/PC ratio in the present study, most of the $\mathrm{HA}$ was bound to $\mathrm{PC}$ vesicles at $\mathrm{pH}$ 7.4 (Fig. $3 A$ ). The partition coefficient $\mathrm{K}_{\mathrm{p}}$ for $\mathrm{HA}$ is 0.03 , an order of magnitude greater than that of oleic acid, reflecting a much lower affinity of BSA for HA. With a threefold excess of BSA, most of the fatty acid was bound to BSA (Fig. $3 C$ ), as predicted by the $K_{p}$.

The decrease in the relative affinity of BSA for HA compared to membranes may be a result both of a higher affinity of PC vesicles for HA and a lower affinity of BSA for HA in comparison to oleic acid. The latter possibility is suggested by the observation of only one structurally distinct binding site by NMR. Previous equilibrium binding studies suggested decreased binding of VLCFA to albumin [42]; the maximum 
molar ratio obtained with $\mathrm{C} 22: 0$ was 0.5 , compared with up to 10.0 (for laurate, $\mathrm{C} 12: 0$ ). We observed a maximum binding of $\sim 1$ mole of HA per mole of BSA (Fig. $3 C$ ). Long chain fatty acids (12-18 carbons) bind to multiple high-affinity sites with similar affinities, enhancing their binding capacity, and show 2-3 carboxyl resonances representing these binding sites (19$21,24,25$ ). The chemical shift for HA bound to BSA does not correspond to any of the chemical shifts for high affinity sites but to lower affinity sites $(19,20,24)$, suggesting that the very long chain of HA prevents it from binding in any of the highaffinity sites. We therefore predict that the binding site structure and affinity of serum albumin will promote higher partitioning of HA into membranes, and that albumin will not function to alleviate undesired high concentrations of HA in membranes, as it does with typical fatty acids. The lower affinity of albumin therefore is likely to exacerbate the effects of HA on membrane structure and membrane components.

pH-dependent partitioning. Compared with other fatty acids, HA not only showed similar ionization behavior in phospholipid bilayers (as discussed above), but the partitioning between BSA and PC vesicles was also similarly $\mathrm{pH}$ dependent. Slightly lower than physiological $\mathrm{pH}$ increased partitioning of HA from BSA to vesicles (Fig. 4). This finding conforms to previous results with shorter chain fatty acids showing that the uncharged, relative to the charged, form of fatty acid binds more favorably to the phospholipid bilayer (43). The pH-dependent experiments also illustrated the free and reversible movement of HA between vesicles and albumin, a result consistent with the hypothesis of simple physico-chemical partitioning of fatty acids between albumin and membranes $(22,32)$. Therefore, we predict that in vivo HA can partition between membranes and serum albumin without mediation by coupling to any energy dependent processes, and with enhanced partitioning to the membrane under acidic $\mathrm{pH}$ conditions.

Role of VLCFA excess in the pathogenesis of ALD. The studies presented here indicate that an excess of VLCFA, such as that observed in ALD, can have a profound effect on membrane structure, and that the rate of removal of VLCFA from the membrane may be several orders of magnitude slower than the rates of the more common long chain fatty acids. While our studies were conducted in artificial membrane systems, the observations that membrane microviscosity is increased in red blood cells of ALD patients (16) and that such an increase is associated with impaired ACTH responsiveness in cultured adrenocortical cells (17) suggest that our results are relevant to natural membrane systems.

It thus appears likely that accumulation of VLCFA does have a deleterious effect on membrane structure and function, but it is not yet possible to define the exact role of VLCFA in the pathogenesis of ALD. Correlative studies in postmortem suggested that the adrenocortical insufficiency was caused by the accumulation of cholesterol esterified with VLCFA (7). Ogino et al. (44) have shown that such esters are poor substrates for cholesterol hydrolases, and that this contributes both to their accumulation and to the impaired capacity of the adrenal gland to hydrolyze cholesterol esters in response to ACTH stimulation. (The decreased hydrolysis of cholesteryl esters with VCLFA could be explained by an inability to partition into the interface where hydrolysis occurs [45].) The adrenal pathology of ALD thus may be a consequence both of deleterious effects of VLCFA on membrane structure, and the accumulation of the abnormal cholesterol esters.

The pathogenesis of the nervous system pathology in ALD is complex, and recent studies indicate that it involves at least two mechanisms. Patients with the most severe form of the disease exhibit an acute inflammatory demyelinating process that resembles multiple sclerosis and involves the action of cytokines such as tumor necrosis factor alpha (8) and possibly autoimmune mechanisms (9). Segregation analysis suggests the action of an autosomal modifier gene (46) which is postulated to modulate the extent of a cytokine or immune-mediated cascade triggered in some way by the VLCFA excess. This "trigger" may be related to a disruption of the membrane structure by VLCFA, or perhaps more likely, VLCFA release may lead to an inflammatory response via cytokine or immune-mediated mechanisms.

As already noted, this inflammatory response is mild or absent in patients with AMN, who survive to adulthood, and in whom the pathology is mainly axonal and involves the distal aspects of the long tracts of the spinal cord (47). Theda et al. (39) have shown that an excess of HA in the phosphatidylcholine fraction appears to be the earliest biochemical abnormality in ALD brain tissue. We speculate that this excess of HA alters nervous system cell membrane function and leads to the progressive axonal dysfunction associated with AMN. Further definition of this postulated pathogenetic mechanism may be aided by in vitro studies of neuronal and glial cultures, although such studies have been hampered so far by the very low solubility of HA.

The implication of the present study that HA may have profound effects on membrane structure suggests that it is warranted to continue efforts to lower HA levels in the nervous system of ALD patients. Recent studies suggest that the limited clinical benefit of "Lorenzo's Oil" is attributable to the failure of erucic acid, the active ingredient of this oil, to enter the nervous system in significant quantity $(48,49)$. It is hoped that reduction of brain VLCFA can be achieved by bone marrow transplantation (50) and eventually gene therapy approaches that can be tested in transgenic animal models of ALD now being developed.

\section{Acknowledgments}

This was supported by grants HL-26335 (J. A. Hamilton), and NS13559 (Y. Kishimoto). The authors wish to thank Dr. Amir Salmon for purification of the ${ }^{13} \mathrm{C}$-hexacosanoic acid, and Margaret Gibbons for preparation of the manuscript.

\section{References}

1. Moser, H. W., and A. B. Moser. 1989. X-linked adrenoleukodystrophy. In The Metabolic Basis of Inherited Disease. C. R. Scriver, A. L. Beaudet, W. S. Sly, and D. Valle, editors. McGraw Hill, New York, Sixth Edition. 1511-1532.

2. Poulos, A. 1995. Very long chain fatty acids in higher animals - A review. Lipids 30:1-14.

3. Lazo, O., M. Contreras, M. Hashmi, W. Stanley, C. Irazu, and I. Singh. 1988. Peroxisomal lignoceroyl-CoA ligase deficiency in childhood adrenoleukodystrophy and adrenomyeloneuropathy. Proc. Natl. Acad. Sci. USA. 85:76477651 .

4. Wanders, R. J., C. W. Van Roermund, M. J. van Wijland, R. B. Schutgens, H. van den Bosch, A. W. Schram, and J. M. Tager. 1988. Direct evidence that the deficient oxidation of very long chain fatty acids in X-linked adrenoleukodystrophy is due to an impaired ability of peroxisomes to activate very long chain fatty acids. Biochem. Biophys. Res. Commun. 153:618-624.

5. Singh, I., A. B. Moser, S. Goldfischer, and H. W. Moser. 1994. Lignoceric acid is oxidized in the peroxisomes: implications for the Zelleger cerebro-hepatorenal syndrome and adrenoleukodystrophy. Proc. Natl. Acad. Sci. USA. 81:42034207.

6. Mosser, J., A. M. Douar, C. O. Sarde, P. Kioschis, R. Feil, H. Moser, A. M. Poustka, J. M. Mandel, and P. Aubourg. 1993. Putative X-linked adrenoleukodys- 
trophy gene shares unexpected homology with $\mathrm{ABC}$ transporter. Nature (Lond.) 361:726-730.

7. Powers, J. M., H. H. Schaumburg, A. B. Johnson, and C. S. Raine. 1980 A correlative study of the adrenal cortex in adrenoleukodystrophy: Evidence for a fatal intoxication with very long chain fatty acids. Invest. Cell Pathol. 3:353376.

8. Powers, J. M., Y. Liu, A. Moser, and H. W. Moser. 1992. The inflammatory myelinopathy of adreno-leukodystrophy: Cells, effector molecules, and pathogenetic implications. J. Neuropathol. Exp. Neurol. 51:630-643.

9. Griffin, D. E., H. W. Moser, Q. Mendoza, T. Moench, S. O'Toole, and A. B. Moser. 1985. Identification of the inflammatory cells in the nervous system of patients with adrenoleukodystropy. Ann. Neurol. 18:660-664.

10. Moser, H. W., F. Kok, S. Neumann, J. Borel, A. Bergin, S. Darwish Mostafa, R. Panoscha, C. T. Davoili, J. Shankroff, and K. D. Smith. 1994. Adrenoleukodystrophy update: Genetics and effect of Lorenzo's oil therapy in asymptomatic patients. Int. J. Pediatr. 9:196-204.

11. Rizzo, W. B., R. T. Leshner, A. Odone, D. A. Craft, S. S. Jennings, R. Jaitly, and J. A. Segro. 1990. X-linked adrenoleukodystropy: Biochemical and clinical efficacy of dietary erucic acid therapy. In: Adrenoleukodystrophy and other peroxisomal disorders. G. Uziel, R. J. A. Wanders, and M. Cappa editors Excerpta Medica, International Congress Series 898, Amsterdam. 149-162.

12. Odone, A., and M. Odone. 1989. Lorenzo's oil. A new treatment for adrenoleukodystrophy. J. Pediatr. Neurosci. 5:55-61.

13. Moser H. W., A. B. Moser, K. D. Smith, A. Bergin, J. Borel, J. Schankroff, O. C. Stine, C. Merette, J. Ott, W. Krivit, and E. Shapiro. 1992. Adrenoleukodystrophy: phenotypic variability: Implications for therapy. J. Inher. Metab. Dis. 15:645-664.

14. Aubourg, P., C. Adamsbaum, M. C. Lavallard-Rousseau, F. Rocchiccioli, N. Cartier, I. Jambaque, C. Jakobezak, A. Lemaitre, F. Boureau, C. Wolf, and P. F. Bougneres. 1993. A two-year trial of oleic and erucic acids ("Lorenzo's oil") as treatment for adrenomyeloneuropathy. N. Engl. J. Med. 329:745-752.

15. Moser, H. W. S. Newmann, A. Bergin, S. D. Mostava, R. Panoscha C. T. Davoli, J. Shankroff, and K. D. Smith. 1994. Adrenoleukodystrophy update: Genetics and effect of Lorenzo's Oil therapy in asymptomatic patients. Interna tional Pediatrics. 9:196-204.

16. Knazek, R. A. W. B. Rizzo, J. D. Schulman, and J. R. Dave. 1983. Membrane microviscosity is increased in the erythrocytes of patients with adrenoleukodystrophy and adrenomyeloneuropathy. J. Clin. Invest. 72:245-248.

17. Whitcomb, R. W., W. M. Linehan, and R. A. Knazek. 1988. Effects of long-chain, saturated fatty acids on membrane microviscosity and adrenocorticotropin responsiveness of human adrenocortical cells in vitro. J. Clin. Invest. 81:185-188.

18. Kantor, H. L., and J. Prestegard. 1978. Fusion of phosphatidylcholine bilayer vesicles: role of free fatty acid. Biochemistry. 17:3592-3597.

19. Parks, J. S., D. P. Cistola, D. M. Small, and J. A. Hamilton. 1983 Interactions of the carboxyl group of oleic acid with bovine serum albumin: ${ }^{13} \mathrm{C}$ NMR study. J. Biol. Chem. 258:9262-9269.

20. Hamilton, J. A. 1992. Binding of fatty acids to serum albumin. NIPS 7:9264-270

21. Hamilton, J. A. 1994. Studies of fatty acids by high resolution and solid state ${ }^{13} \mathrm{C}$ NMR spectroscopy interactions with phospholipid bilayers and proteins. In Carbon-13 NMR Spectroscopy of Biological Systems. N. Beckmann, editor. Academic Press, New York. 117-157.

22. Hamilton, J. A., and D. P. Cistola. 1986. Transfer of oleic acid between albumin and phospholipid. Proc. Natl. Acad. Sci. USA. 83:82-86.

23. Hamilton, J. A. 1989. Medium-chain fatty acid binding to albumin and transfer to phospholipid bilayers. Proc. Natl. Acad. Sci. USA. 86:2663-2667.

24. Cistola, D. P., D. M. Small, and J. A. Hamilton. 1987. Carbon-13 NMR studies of saturated fatty acids bound to bovine serum albumin. I. The filling of individual fatty acid binding sites. J. Biol. Chem. 262:10971-10979.

25. Cistola, D. P., D. M. Small, and J. A. Hamilton. 1987. Carbon-13 NMR studies of saturated fatty acids bound to bovine serum albumin. II. Electrostatic Interactions in individual fatty acid binding sites. J. Biol. Chem. 262:1098010985.

26. Schullery, S. E., T. A. Seder, D. A. Weinstein, and D. A. Bryant. 1981 Differential thermal analysis of dipalmitoylphosphatidylcholine-fatty acid mixtures. Biochemistry. 20:6818-6824.

27. Eliasz, A. W., D. Chapman, and D. F. Ewing. 1976. Phospholipid phase transitions. Effects of n-alcohols, n-monocarboxylic acids, phenylalkyl alcohols and quaternary ammonium compounds. Biochim. Biophys. Acta. 448:220-230.

28. Jain, M. K., and N. Min Wu. 1977. Effects of small molecules on the dipalmitoyl lecithin liposomal bilayer: III. Phase transition in lipid bilayer. Membrane Biol. 34:157-201.

29. Murad, S., R. H. Chen, and Y. Kishimoto. 1977. Alpha-hydroxylation of fatty acids in brain. Substrate specificity and deuterium isotope effect. J. Biol. Chem. 252:5206-5210.

30. Ruocco, M. J., and G. G. Shipley. 1982. Characterization of the subtransition of hydrated dipalmitoylphosphatidylcholine bilayers: $x$-ray diffraction study. Biochim. Biophys. Acta 684:59.

31. Spector, A. A. 1975. Fatty acid binding to plasma albumin. J. Lipid Res. 16:165-179.

32. Daniels, C., N. Noy, and D. Zakim. 1985. Rates of hydration of fatty acids bound to unilamellar vesicles of phosphatidylcholine or to albumin. Biochemistry. 24:3286-3292.

33. Lund-Katz, S., H. M. Laboda, L. R. McLean, and M. C. Phillips. 1988. Influence of molecular packing and phospholipid type on rates of cholesterol exchange. Biochemistry. 27:3416-3423.

34. Kamp F., and J. A. Hamilton. 1992. pH gradients across phospholipid membranes caused by fast flip-flop of un-ionized fatty acids. Proc. Natl. Acad. Sci. USA. 89:11367-11370.

35. Ordway, R. W., J. V. Walsh, Jr., and J. J. Singer. 1989. Arachidonic acid and other fatty acids directly activate potassium channels in smooth muscle cells. Science (Wash. DC). 244:1176-1179.

36. Huang, J. M.-C., H. Xian, and M. Bacaner. 1992. Long-chain fatty acids activate calcium channels in ventricular myocytes. Proc. Natl. Acad. Sci. USA. 89:6452-6456.

37. Nishizuka, Y. 1992. Intracellyular signaling by hydrolysis of phospholipids and activation of protein kinase C. Science (Wash. DC). 258:607-614.

38. Hamilton, J. A., V. N. Civelek, F. Kamp, K. Tornheim, and B. E. Corkey. 1994. Changes in internal pH caused by movement of fatty acids into and out of clonal pancreatic B-cells (HIT). J. Biol. Chem. 269:10852-10856.

39. Theda, C., A. B. Moser, J. M. Powers, and H. W. Moser. 1992. Phospholipids in X-linked adrenoleukodystrophy white matter: Fatty acid abnormalities before the onset of demyelination. J. Neurol. Sci. 110(1-2):195-204.

40. Brown, F. R., III, W. W. Chen, D. A. Kirschner, K. L. Frayer, J. M. Powers, A. B. Moser, and H. W. Moser. 1983. Myelin membrane from adrenoleukodystrophy brain white matter - Biochemical properties. J. Neurochem. 41:341 348.

41. Bizzozero, O. A., G. Zuniga, and M. B. Lees. 1991. Fatty acid composition of human myelin proteolipid protein in peroxisomal disorders. J. Neurochem. $56: 872-878$.

42. Spector, A. A., and J. C. Hoak. 1969. An improved method for the addition of long-chain free fatty acid to protein solutions. analyt. Biochemistry. 32:297302.

43. Peitzsch, R. M., and S. McLaughlin. 1993. Binding of acylated peptides and fatty acids to phospholipid vesicles: Pertinence to myristoylated proteins. Biochemistry. 32:10436-10443.

44. Ogino, T., and K. Suzuki. 1981. Specificities of human and rat brain enzymes of cholesterol ester metabolism toward very long chain fatty acids: Implication for biochemical pathogenesis of adrenoleukodystrophy. J. Neurochem. 36:776-779.

45. Hamilton, J. A., and D. M. Small. 1982. Solubilization and localization of cholesteryl oleate in egg phosphatidylcholine vesicles. J. Biol. Chem. 257:7318-7321.

46. Watts, R. W., S. H. Morgan, C. J. Danpure, P. Purkiss, R. Y. Calne, K. Rolles, L. R. Baker, M. A. Mansell, L. H. Smith, and R. M. Merion. 1991. Combined hepatic and renal transplantation in primary hyperoxaluria type I: Clinical report of nine cases. Am. J. Med. 90:179-188.

47. Power, J. M. 1985. Adrenoleukodystrophy (Adreno-testiculo-leuko-neuropathic-complex ). Clin. Neuropathol. 4:181-199.

48. Rasmussen, M., A. B. Moser, J. Borel, and H. W. Moser. 1994. Brain erucic and very long chain fatty acid levels in adrenoleukodystrophy patients treated with glyceryl trioleated and trierucate oils (Lorenzo's oil). Neurochem. Res. 19:1073-1082.

49. Poulos, A., R. Gibson, P. Sharp, K. Beckman, and P. Grattan-Smith. 1994. Very long chain fatty acids in X-linked adrenoleukodystrophy brain after treatment with Lorenzo's oil. Ann. Neurol. 36:741-746.

50. Auborg, P., S. Blanche, I. Jambaque, F. Rocchiccioli, G. Kalifa, C. NaudSaudreau, M. O. Rolland, M. Debre, J. L. Chaussain, C. Griscelli, A. Fischer, and P. F. Bougnere. 1990. Reversal of early neurologic and neuroradiologic manifestation of X-linked adrenoleukodystrophy by bone marrow transplantation. N. Engl. J. Med. 322:1860-1866. 